\title{
Audit of NICE compliance with warfarin for non-valvular atrial fibrillation on admission to healthcare for older people wards in a university teaching hospital
}

\author{
Authors: Emily Little ${ }^{\mathrm{A}}$ and Rajesh Dwivedi ${ }^{\mathrm{A}}$
}

\section{Introduction}

Traditionally warfarin has been mainly used for stroke thromboprophylaxis in patients with atrial fibrillation, but direct oral anticoagulants (DOACs) are increasingly being used as they have improved safety profiles with comparable efficacy. Recent data from the National Reporting and Learning System have uncovered safety incidents related to anticoagulation, predominantly warfarin, contributing in excess of $10 \%$ of all medication errors. ${ }^{1}$ Our department recently witnessed a number of safety incidents including high-level investigation related to anticoagulation, predominantly warfarin. The National Institute for Health and Care Excellence (NICE) recommends reassessing anticoagulation choice for patients on warfarin with poor anticoagulation control, indicated by any of the following:

$>$ two international normalised ratio (INR) values $<1.5$ or $>5$ or one INR value $>8$, within the past 6 months

$>$ Time in therapeutic range (TTR) $<65 \%$.

\section{Materials and methods}

Eighty-eight patients on warfarin were initially screened between August and December 2018. After excluding younger patients ( $<65$ years), warfarin for any other indication etc, 50 patients were included in the audit. Data were collected retrospectively through review of medical notes on the DHR (digital health records) and review of their INR over the previous 6 months to record any significant deviations outside of the therapeutic range as per NICE guidance. TTR over the past 6 months was calculated using the Rosendaal method.

\section{Results and discussion}

The median age was 83 years. $29 / 50(58 \%)$ patients had TTR $<65 \%$; 32/50 (64\%) patients had unstable INRs; 31/50 (62\%) patients had warfarin continued on discharge despite TTR $<65 \%$ or unstable INRs. Only 11 patients had both TTR
$>65 \%$ and stable INRs as per NICE guidance. Importantly, $39 / 50(78 \%)$ should have DOAC instead of warfarin as per NICE guidance.

\section{Conclusion}

Our study demonstrates poor compliance with NICE guidance regarding warfarin for non-valvular atrial fibrillation in older patients. Reasons for this include polypharmacy, comorbidities, illness, malnutrition etc. We found that the majority of older patients on warfarin should be offered DOAC instead as they offer improved safety profiles with comparable efficacy. The results of this audit have been presented and widely disseminated to our department. A re-audit is planned within the next 6 months to assess improvement.

We suggest that geriatricians proactively review the appropriateness of warfarin prescription and consider a DOAC in preference to warfarin in all older patients.

\section{Conflicts of interest}

None declared.

\section{Reference}

1 Cousins D. A review of medication incidents reported to the National Reporting and Learning System in England and Wales over 6 years (2005-2010). Br J Clin Pharmacol 2012;74:597-604. 